\title{
Internal Resonance of Hyperelastic Thin-Walled Cylindrical Shells under Harmonic Axial Excitation and Time-Varying Temperature Field
}

Jie Xu

Dalian University of Technology

Yuan Xuegang ( $\square$ yxg1971@163.com)

Dalian Minzu University: Dalian Nationalities University https://orcid.org/0000-0003-4719-364X

Hongwu Zhang

Dalian University of Technology

Minfu Ma

Dalian Minzu University: Dalian Nationalities University

Wei Zhao

Dalian Minzu University: Dalian Nationalities University

\section{Research Article}

Keywords: Hyperelastic cylindrical shell, axisymmetric nonlinear vibration, harmonic balance method, internal resonance, isolated bubble

Posted Date: August 17th, 2021

DOI: https://doi.org/10.21203/rs.3.rs-761448/v1

License: (c) (1) This work is licensed under a Creative Commons Attribution 4.0 International License.

Read Full License

Version of Record: A version of this preprint was published at Thin-Walled Structures on June 1st, 2022. See the published version at https://doi.org/10.1016/j.tws.2022.109256. 


\section{Internal resonance of hyperelastic thin-walled \\ 2 cylindrical shells under harmonic axial \\ 3 excitation and time-varying temperature field}

4 Jie Xu ${ }^{2} \cdot$ Xuegang Yuan ${ }^{1,2 *} \cdot$ Hongwu Zhang $^{2} \cdot$ Minfu Ma $^{1,3} \cdot$ Wei Zhao ${ }^{1,4}$

5 1. X. Yuan $\cdot$ M. Ma $\cdot$ W. Zhao

6 School of Science, Dalian Minzu University, Dalian 116600, P. R. China

7 2. J. Xu $\cdot$ H. Zhang

8 State Key Laboratory of Structural Analysis for Industrial Equipment, Department

9 of Engineering Mechanics, Faculty of Vehicle Engineering and Mechanics,

10 Dalian University of Technology, Dalian 116024, P. R. China

11 3. M. Ma

12 College of Mathematics and Information, North Minzu University, Yinchuan

13 750021, P. R. China

14 4. W. Zhao

15 Beijing Key Laboratory of Nonlinear Vibrations and Strength of Mechanical

16 Structures, College of Mechanical Engineering, Beijing University of

17 Technology,Beijing 100124, P. R. China

18 Corresponding author: Xuegang Yuan, e-mail: yxg1971@163.com

19 Abstract In this paper, the internal resonance characteristics of hyperelastic cylindrical shells

20 under the time-varying temperature field are investigated for the first time, and the evolution of the

21 isolated bubble is carried out. Through the analysis of the influences of temperature on material

22 parameters, the hyperelastic strain energy density function in the unsteady temperature field is

23 presented. The governing equations describing the axisymmetric nonlinear vibration are derived

24 from the nonlinear thin shell theory and the variational principle. With the harmonic balance

25 method and the arc length method, the steady state solutions of shells are obtained, and their

26 stabilities are determined. The influences of the discrete mode number, structural and temperature

27 parameters on the nonlinear behaviors are examined. The role of the parameter variation in

28 evolution behaviors of isolated bubble responses is revealed under the condition of 3:1 internal

29 resonance. The results manifest that both structural and temperature parameters can affect the

30 resonance range of the response curve, and the perturbed temperature has a more significant effect

31 on the stable region of the solution.

32 Keywords Hyperelastic cylindrical shell · axisymmetric nonlinear vibration .

33 harmonic balance method $\cdot$ internal resonance $\cdot$ isolated bubble 


\section{$1 \quad 1$ Introduction}

2 Due to the light weight, high strength and stiffness ratio, cylindrical shells are

3 widely applied in many important engineering fields, such as the energy

4 transportation, shipbuilding and aerospace. The pipeline conveying fluid and the

5 spacecraft or missile, both can be modeled as the cylindrical shell for simplifying

6 analysis ${ }^{[1-3]}$. In addition, the mechanical behavior of biological tissues such as

7 bones and blood vessels can also be simulated with the cylindrical shell. However,

8 it should be noted that for those structures, hyperelastic material models should be

9 adopted to describe the nonlinear mechanical behaviors ${ }^{[4,5]}$. Generally,

10 cylindrical shells are usually subjected to different loads (such as the axial force

11 and temperature), which are prone to induce the large amplitude vibration, and

12 then destroy the structural safety. Therefore, it is essential and necessary to

13 investigate the nonlinear vibration characteristics of hyperelastic thin-walled

14 cylindrical shells subjected to external loads for the optimization of design and 15 improvement of safety.

16 The thin-walled shell is usually the best and only choice for those aerospace 17 and navigation structures, as well as engineering structures such as vehicle and 18 architecture building. Due to the complexity of the vibration behavior of the 19 continuum structure, the discretization method is widely applied to simplify the 20 continuum into a system with finite degree of freedom. This method is also very 21 common in the vibration analysis of beams, and there is a solid theoretical 22 foundation for the related analysis methods. However, shells especially exhibit 23 certain effects that are not presented in beams or even plates, and cannot be 24 interpreted by engineers who are only familiar with the beam-type vibration 25 theory $^{[6]}$. Thus, it is helpful to improve the understanding of basic phenomena in 26 vibrations of plates and shells, and it will be useful in explaining experimental 27 measurements or the results of the finite element programs. Radwanska et al. ${ }^{[7]}$ 28 presented a comprehensive introduction to the elastic plate and shell theory, 29 formulations and solutions of fundamental mechanical problems (statics, stability 30 and free vibrations) using the exact approaches and approximate computational 31 methods, and also emphasized the modern capabilities of the finite element 32 technology. With the load increases, the shell behavior will show some essential 33 geometric nonlinearity who plays a key role in the structural safety. Therefore, the 
1 influence of nonlinearity has also been paid more and more attention in the 2 dynamic analysis of thin-walled shells. Meanwhile, with the development of 3 material science, the combination between shells and new materials is getting 4 more and more inseparably associated, especially, for plates and shells composed 5 of composite materials. The monograph contributed by Reddy ${ }^{[8]}$ systematically 6 introduced various aspects related to laminated composite structures, including the 7 virtual work principle, variational method, anisotropic elasticity, plate and shell 8 theory (including classical theory, first-order and third-order shear deformation 9 theory), geometric nonlinearity and finite element analysis. Shen ${ }^{[9,10]}$ dedicated to 10 the investigation of the geometrically nonlinear problems of inhomogeneous 11 isotropic and functionally graded plates and shells, which includes the large 12 deflection, post-buckling and nonlinear vibration. Amabili ${ }^{[11]}$ studied the plates 13 and shells composed of composite materials, soft materials and biological 14 materials, and researched the hyperelastic, viscoelasticity and nonlinear damping, 15 which are pioneering works considered both material and geometric 16 nonlinearities.

17 The researches on nonlinear dynamic behaviors of cylindrical shells have 18 attracted much attention from a large number of scholars. Ye and Wang [12] 19 analyzed the nonlinear forced vibration of the functionally graded graphene 20 platelet-reinforced metal foam thin-walled cylindrical shell, and found that the 21 inclusion of graphene platelets in the shells weakens the nonlinear coupling effect.

22 Based on the von Kármán geometric nonlinear strain-displacement relationship 23 and the first-order shear deformation theory, Zhang et al. ${ }^{[13]}$ studied the nonlinear 24 radial breathing vibration of a carbon fiber reinforced polymer laminated 25 cylindrical shell with different temperatures under both axial pressure and radial 26 line load. Yang et al. ${ }^{[14]}$ studied the nonlinear vibration of a carbon fiber 27 reinforced polymer laminated cylindrical shell with 1:2 internal resonance, 28 primary parametric resonance and 1/2 subharmonic resonance. Based on Reddy's 29 third order shear deformation theory and the Galerkin method, Vuong and Duc ${ }^{[15]}$ 30 examined the nonlinear vibration of the functionally graded moderately thick 31 toroidal shell segments resting on the Pasternak type elastic foundation. Liu et al. $32{ }^{[2]}$ investigated the nonlinear breathing vibration of an eccentric rotating 33 composite laminated cylindrical shell subjected to the lateral and temperature 
1 excitations. Parvez et al. ${ }^{[16]}$ presented the nonlinear dynamic response of

2 laminated composite cylindrical shells under periodic external forces, and

3 explored the parameters influencing the transition between the softening and

4 hardening nonlinear behaviors of cylindrical shells. Shen et al. ${ }^{[17]}$ investigated the

5 nonlinear flexural vibrations of carbon nanotube-reinforced composite laminated

6 cylindrical shells with negative Poisson's ratios in thermal environments. Wang et

7 al. ${ }^{[18]}$ investigated the strongly nonlinear traveling waves in a thermo-hyperelastic 8 cylindrical shell.

9 Compared with those structures composed of composite materials or other new 10 materials, there are relatively few researches on the dynamics of hyperelastic 11 structures, and most of them are also focused on relatively simple structures, such 12 as the membrane ${ }^{[19-21]}$ or the beam ${ }^{[22-24]}$ contributed by Soares, Gonçalves, Zhao, 13 Chen and Wang. Dong et al. ${ }^{[25]}$ proposed a novel approach to tune the resonance 14 frequency of circular hyperelastic membrane-based energy harvesters via different 15 stretch ratios applied to membranes, which provided an alternative tuning strategy 16 to enable energy harvesting from different ambient vibration sources in various 17 environments. Wang and Zhu ${ }^{[26]}$ investigated the nonlinear vibrations of a 18 hyperelastic beam under time-varying axial loading are derived via the extended 19 Hamilton's principle. Iglesias et al. ${ }^{[27]}$ studied the large-amplitude axisymmetric 20 free vibration of an incompressible hyperelastic orthotropic cylinder, and analyzed 21 the influence of initial conditions, structural and material parameters on the 22 dynamic behavior. With the Gent-Gent hyperelastic model, Alibakhshi and 23 Heidari ${ }^{[28]}$ researched the nonlinear vibration of a dielectric elastomer balloon 24 considering the strain hardening and the second invariant of the Cauchy-Green 25 deformation tensor, and found that the second invariant parameter could suppress 26 the chaotic motion of the system. Due to the existence of geometric and material 27 nonlinearities, it is difficult to investigate the vibrations of hyperelastic plate and 28 shell structures, however, there are still a few related works. Breslavsky et al. ${ }^{\text {[29] }}$ 29 presented the static and dynamic responses of circular cylindrical shells composed 30 of hyperelastic arterial materials based on the nonlinear high-order shear 31 deformation theory, and pointed out that the resonant regime with both driven and 32 companion modes active should possess more complicated nonlinear dynamics. 33 Amabili et al. ${ }^{[30]}$ conducted the numerical analysis and experimental research on 
1 the hyperelastic behavior of a thin square silicone rubber plate, obtained a good agreement between the first four natural modes and frequencies numerically and experimentally.

4 It is not difficult to find that there are numerous researches on the nonlinear dynamics of cylindrical shells, however, few researchers pay attention to the

6 dynamic behaviors of hyperelastic cylindrical shells considering the material

7 nonlinearity, and fewer works considering the temperature effect. Therefore, this 8 paper studies the nonlinear dynamic behaviors of the hyperelastic thin-walled

9 cylindrical shells under harmonic axial excitation and time-varying temperature 10 field. The remainder of this paper is organized as follows: Section 2 introduces the 11 thermo-hyperelastic constitutive relationship; Section 3 gives the strain12 displacement relationship of the thin-walled cylindrical shell, and derives the 13 nonlinear governing differential equations; Section 4 analyzes the natural 14 frequency characteristics of the shells and calculates the corresponding internal 15 resonance parameters; Section 5 presents the solution of nonlinear equations and 16 the stability of periodic solutions; Section 6 discusses the influences of the 17 discrete mode number, structural and temperature parameters via the numerical 18 simulation; Section 7 draws the conclusions.

\section{2. Thermo-hyperelasticity and strain-displacement 20 relationship}

21 Consider a thin-walled cylindrical shell composed of a class of incompressible 22 thermo-hyperelastic materials in a time-varying temperature field, which is 23 subjected to the axial load at both ends. As shown in Fig. 1, the length, diameter 24 of the middle surface and thickness of the cylindrical shell are represented by $H$, $25 D$ and $h$, respectively. The cylindrical coordinate system $(x, \theta, z)$ is 26 established on the mid-surface of the shell, where $x, \theta, z$ are the axial, 27 circumferential and radial directions. Let $u, v$ and $w$ be the displacements of 28 a point at the mid-surface, and $u_{1}, u_{2}$ and $u_{3}$ be the displacements of an 29 arbitrary point of the shell accordingly. 


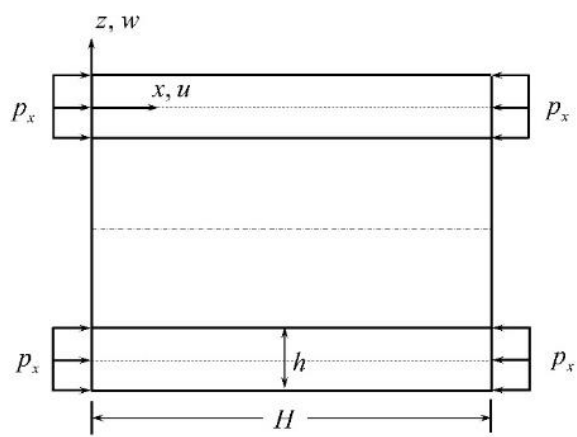

Fig. 1 Cylindrical shell configuration under periodic axial loads

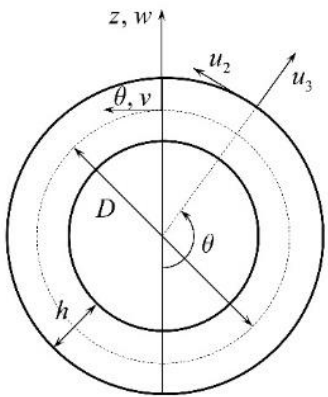

7 where $\varepsilon_{i j}(i, j=1 \sim 3)$ is the Green strain. The principal invariants of the right

8 Cauchy-Green deformation tensor are

$$
I_{1}=\operatorname{tr}(\mathbf{C})=2\left(\varepsilon_{11}+\varepsilon_{22}+\varepsilon_{33}\right)+3,
$$

$$
\begin{aligned}
& I_{2}=\frac{1}{2}\left[(\operatorname{tr} \mathbf{C})^{2}-\operatorname{tr}\left(\mathbf{C}^{2}\right)\right]=4\left(\varepsilon_{11}+\varepsilon_{22}+\varepsilon_{33}+\varepsilon_{11} \varepsilon_{22}+\varepsilon_{11} \varepsilon_{33}+\varepsilon_{22} \varepsilon_{33}\right)-\varepsilon_{12}^{2}+3, \\
& I_{3}=J^{2}=\operatorname{det}(\mathbf{C})=\left(2 \varepsilon_{33}+1\right)\left[\left(2 \varepsilon_{11}+1\right)\left(2 \varepsilon_{22}+1\right)-\varepsilon_{12}^{2}\right]
\end{aligned}
$$

10 Using with the incompressible constraint $J=1$ yields

$$
\varepsilon_{33}=\frac{1}{2}\left\{\left[\left(2 \varepsilon_{11}+1\right)\left(2 \varepsilon_{22}+1\right)-\varepsilon_{12}^{2}\right]^{-1}-1\right\}
$$

12 For the case of small strains, expanding Eq. (3) about all the strains in it up to the

13 fourth order gives

$$
\begin{aligned}
\varepsilon_{33}= & -\left(\varepsilon_{11}+\varepsilon_{22}\right)+2\left(\varepsilon_{11}^{2}+\varepsilon_{22}^{2}+\varepsilon_{11} \varepsilon_{22}+\varepsilon_{12}^{2}\right) \\
& -4\left(\varepsilon_{11}+\varepsilon_{22}\right)\left(\varepsilon_{11}^{2}+\varepsilon_{22}^{2}+\frac{1}{2} \varepsilon_{12}^{2}\right)+8\left(\varepsilon_{11}^{4}+\varepsilon_{22}^{4}+\frac{1}{16} \varepsilon_{12}^{4}\right) \\
& +8 \varepsilon_{11} \varepsilon_{22}\left(\varepsilon_{11}^{2}+\varepsilon_{22}^{2}+\varepsilon_{11} \varepsilon_{22}+\varepsilon_{12}^{2}\right)+6 \varepsilon_{12}^{2}\left(\varepsilon_{11}^{2}+\varepsilon_{22}^{2}\right)+\cdots
\end{aligned}
$$

15 Based on the Kirchhoff-love hypothesis (that is, the stress components 16 perpendicular to the mid-surface are negligible, which is a very good 17 approximation for thin-walled shells) and the assumption of axisymmetric 18 vibrations (the circumferential displacement is zero), the displacements of a 19 generic point on the cylindrical shell are given as follows 


$$
u_{1}=u(x, t)-\frac{\partial w(x, t)}{\partial x} z, u_{2}=0, u_{3}=w(x, t)
$$

2 Additionally, the geometrical nonlinearity of the shell yields the following strain-

3 displacement relationships ${ }^{[8]}$

$$
\begin{aligned}
& \varepsilon_{11}=\frac{\partial u}{\partial x}+\frac{1}{2}\left[\left(\frac{\partial u}{\partial x}\right)^{2}+\left(\frac{\partial w}{\partial x}\right)^{2}\right]-\left(\frac{\partial^{2} w}{\partial x^{2}}+\frac{\partial u}{\partial x} \frac{\partial^{2} w}{\partial x^{2}}\right) z+\frac{1}{2}\left(\frac{\partial^{2} w}{\partial x^{2}}\right)^{2} z^{2} \\
& \varepsilon_{22}=\frac{w}{R}+\frac{1}{2}\left(\frac{w}{R}\right)^{2}
\end{aligned}
$$

For incompressible thermo-hyperelastic materials, the thermal effect is mainly

6 reflected in their strain energy functions. In this paper, the 8-chain non-Gaussian

7 network model, i.e. the Arruda-Boyce model ${ }^{[31]}$ is adopted, as follows,

$$
\Phi_{T_{t}}=\mu_{T_{t}} N_{T_{t}}\left(\frac{\vartheta}{\lambda_{\mathrm{m}}} \sqrt{\frac{I_{1}}{3}}+\ln \frac{\vartheta}{\sinh \vartheta}\right)
$$

$$
\begin{aligned}
& \mu_{T_{t}}=n k T_{t}=\frac{T_{t}}{T_{0}\left(1+\alpha_{\mathrm{T}} \Delta T_{t}\right)^{3}} \mu_{0}, n_{T_{t}}=\frac{n_{0}}{\left(1+\alpha_{\mathrm{T}} \Delta T_{t}\right)^{3}}, \\
& n_{0} N_{0}=n_{T_{t}} N_{T_{t}}, N_{T_{t}}=\left(1+\alpha_{\mathrm{T}} \Delta T_{t}\right)^{3} N_{0}, \Delta T_{t}=T_{t}-T_{0}
\end{aligned}
$$

10 where $\alpha_{\mathrm{T}}$ is the linear thermal expansion coefficient, $T_{t}$ is the environmental

11 temperature, $\mu_{T_{t}}$ is the shear modulus at the temperature of $T_{t}$, and $\Delta T=T_{t}-T_{0}$

12 is the temperature difference $\left(T_{0}\right.$ is room temperature, $(\cdot)_{0}$ is the value of a

13 parameter at the temperature of $T_{0},(\cdot)_{T_{t}}$ is the value of a parameter at the

14 temperature of $\left.T_{t}\right), \lambda_{\mathrm{m}}=\sqrt{N_{T_{t}}}$ is the limited stretch ratio of chains, $N_{T_{t}}$ is the

15 average number of segments in a chain, $\vartheta=L^{-1}\left(\sqrt{I_{1} /\left(3 N_{T_{t}}\right)}\right)$ is the inverse

16 Langevin function. For the periodic uniform environmental temperature, the

17 temperature $T_{\mathrm{M}}$ of the thin-walled shell is assumed to be periodic, then $T_{\mathrm{M}}$ is

18 given by

$$
T_{\mathrm{M}}=T_{\mathrm{A}}+\Delta T_{\mathrm{P}} \cos \left(\omega_{\mathrm{T}} t\right)
$$

20 where $T_{\mathrm{A}}$ is the average temperature part of the environmental temperature and $21 \Delta T_{\mathrm{P}}$ is the amplitude of the perturbed temperature part $\left(\alpha_{\mathrm{T}}\right.$ is a very small 22 parameter, and $\alpha_{\mathrm{T}} \Delta T_{\mathrm{P}}$ is also a small quantity). In this case, 


$$
\begin{gathered}
1-\mu_{\mathrm{M}}=\frac{T_{\mathrm{A}}+\Delta T_{\mathrm{P}} \cos \left(\omega_{\mathrm{T}} t\right)}{T_{\mathrm{A}}\left(1+\alpha_{\mathrm{T}} \Delta T_{\mathrm{P}} \cos \left(\omega_{\mathrm{T}} t\right)\right)^{3}} \mu_{T_{\mathrm{A}}}, N_{\mathrm{M}}=\left[1+\alpha_{\mathrm{T}} \Delta T_{\mathrm{P}} \cos \left(\omega_{\mathrm{T}} t\right)\right]^{3} N_{T_{\mathrm{A}}} \\
2 \quad \mu_{\mathrm{M}} \approx\left[1+P_{\mu} \cos \left(\omega_{\mathrm{T}} t\right)\right] \mu_{T_{\mathrm{A}}}, N_{\mathrm{M}} \approx\left[1+P_{N} \cos \left(\omega_{\mathrm{T}} t\right)\right] N_{T_{\mathrm{A}}} \\
3 \quad \frac{\mu_{\mathrm{M}}}{N_{\mathrm{M}}^{n}}=\frac{\mu_{T_{\mathrm{A}}}}{N_{T_{\mathrm{A}}}^{n}} \frac{1+P_{\mu} \cos \left(\omega_{\mathrm{T}} t\right)}{\left[1+P_{N} \cos \left(\omega_{\mathrm{T}} t\right)\right]^{n}} \approx \frac{\mu_{T_{\mathrm{A}}}}{N_{T_{\mathrm{A}}}^{n}}\left[1+\left(P_{\mu}-n P_{N}\right) \cos \left(\omega_{\mathrm{T}} t\right)\right] \\
P_{\mu}=\left(\frac{1}{T_{\mathrm{A}}}-3 \alpha_{\mathrm{T}}\right) \Delta T_{\mathrm{P}}, P_{N}=3 \alpha_{\mathrm{T}} \Delta T_{\mathrm{P}}
\end{gathered}
$$

5 Moreover, the series expansion form for Eq. (7) (keeping the first five terms) is 6 often adopted as follows,

$$
\Phi_{\mathrm{M}}=\mu_{\mathrm{M}} \sum_{i=1}^{5} \frac{C_{i}}{N_{\mathrm{M}}^{i-1}}\left(I_{1}^{i}-3^{i}\right)
$$

8

$$
\Phi_{\mathrm{M}}=\sum_{i=1}^{5} \frac{\mu_{T_{\mathrm{A}}}}{N_{T_{\mathrm{A}}}^{i-1}}\left\{1+\left[P_{\mu}-(i-1) P_{N}\right] \cos \left(\omega_{\mathrm{T}} t\right)\right\} C_{i}\left(I_{1}^{i}-3^{i}\right)
$$

$$
=\mu_{T_{\mathrm{A}}} \sum_{i=1}^{5} \frac{C_{i}}{N_{T_{\mathrm{A}}}^{i-1}}\left(I_{1}^{i}-3^{i}\right)+\cos \left(\omega_{\mathrm{T}} t\right) \mu_{T_{\mathrm{A}}} \sum_{i=1}^{5} \frac{C_{i}}{N_{T_{\mathrm{A}}}^{i-1}}\left[P_{\mu}-(i-1) P_{N}\right]\left(I_{1}^{i}-3^{i}\right)
$$

9 The material parameters required for the calculation are listed in Table 1.

10

Table1. Material parameters ${ }^{[31,32]}$

\begin{tabular}{cc}
\hline Material parameters & Valves \\
\hline$\mu_{0}(\mathrm{MPa})$ & 0.2853 \\
$N_{0}$ & 26.54 \\
$\alpha_{\mathrm{T}}\left(\mathrm{K}^{-1}\right)$ & $3.6 \times 10^{-4}$ \\
$T_{0}(\mathrm{~K})$ & 297 \\
$C_{1}$ & $1 / 2$ \\
$C_{2}$ & $1 / 20$ \\
$C_{3}$ & $11 / 1050$ \\
$C_{4}$ & $19 / 7000$ \\
$C_{5}$ & $519 / 673750$ \\
\hline
\end{tabular}

11 Combined with Eqs.(2), (4) and (14), the specific expression of the strain energy

12 function is obtained. 


\section{3. System of governing equation}

2 The expressions of the kinetic energy and potential energy can be expressed as

3 follows

$$
D_{P}=\frac{1}{2} \rho \int_{-\frac{h}{2}}^{\frac{h}{2}} \int_{0}^{H} \int_{0}^{2 \pi}\left(\dot{u}_{1}^{2}+\dot{u}_{2}^{2}+\dot{u}_{3}^{2}\right) R \mathrm{~d} \theta \mathrm{d} x \mathrm{~d} z,
$$

$$
E_{P}=\int_{-\frac{h}{2}}^{\frac{h}{2}} \int_{0}^{H} \int_{0}^{2 \pi} \Phi R \mathrm{~d} \theta \mathrm{d} x \mathrm{~d} z
$$

5 where $\rho$ is the mass density, $t$ is the time, $\Phi$ is the strain energy function,

6 and the dot denotes the derivative with respect to $t$.

7 The Ritz method is employed to approximate the axial displacement $u$ and the

8 deflection $w$. The boundary conditions should be satisfied at the shell ends with

9 the assumed modes which are axisymmetric. Then, the displacements are

10 expanded by using the eigenmodes as follows

$$
\left\{\begin{array}{l}
u(x, t)=\sum_{m=1}^{M} u_{m 0}(t) \cos \frac{m \pi x}{H}, \\
w(x, t)=\sum_{m=1}^{M} w_{m 0}(t) \sin \frac{m \pi x}{H}
\end{array}\right.
$$

12 where $m$ is the longitudinal half wave number, and $M$ is the truncation order.

13 It should be noted that under the assumption of axisymmetric motion, the

14 circumferential wave numbers is $0, u_{m 0}(t)$ and $w_{m 0}(t)$ are generalized 15 coordinates related to time $t$.

16 In presence of axial harmonic loads acting on the shell, the virtual work of the 17 external force is done by a time-varying axial load $p_{x}(t)$ which is applied at 18 both ends of the shell. It is positive in the $x$ direction, that is, $-p_{x}(t)$ is applied 19 at $x=0$ and $p_{x}(t)$ is applied at $x=H$. Thus, the uniform axial load $q_{x}(t)$ 20 is expressed as follows

$$
q_{x}(x, \theta, z, t)=p_{x}(t)[-\delta(x)+\delta(x-H)], F_{x}(t)=2 \pi R h p_{x}(t)
$$

22 where $\delta$ is the Dirac delta function, and $F_{x}(t)$ is the net force at the end.

23 Therefore, the virtual work done by the external force can be expressed as

$$
W_{\mathrm{ex}}=\int_{0}^{H} \int_{0}^{2 \pi} \int_{-h / 2}^{h / 2}\left(q_{\mathrm{x}} u+q_{\theta} v+q_{\mathrm{r}} w\right) \mathrm{d} x R \mathrm{~d} \theta \mathrm{d} z=-F_{\mathrm{x}}(t) \sum_{m=1}^{M} u_{m 0}(t)\left[-1+(-1)^{m}\right]
$$


1 The other part of the virtual work is done by the non-conservative damping force.

2 Assuming that the non-conservative damping force is viscous type, Rayleigh's

3 dissipation function is adopted to obtain the virtual work

$$
\begin{aligned}
W_{d} & =\frac{1}{2} \int_{-\frac{h}{2}}^{\frac{h}{2}} \int_{0}^{H} \int_{0}^{2 \pi}\left(c_{1 j} \dot{u}^{2}+c_{2 j} \dot{v}^{2}+c_{3 j} \dot{w}^{2}\right) R \mathrm{~d} \theta \mathrm{d} x \mathrm{~d} z \\
& =\pi h R \int_{0}^{H}\left(c_{1 j} \dot{u}^{2}+c_{2 j} \dot{v}^{2}+c_{3 j} \dot{w}^{2}\right) \mathrm{d} x
\end{aligned}
$$

5 where $c_{i j}$ is damping parameter related to the natural frequency of the shell and

6 can be evaluated from experiments. The Lagrange equation describing the

7 axisymmetric motion for a hyperelastic thin-walled cylindrical shell is given by

$$
\frac{\mathrm{d}}{\mathrm{d} t} \frac{\partial\left(D_{P}-E_{P}\right)}{\partial \dot{q}_{i}}-\frac{\partial\left(D_{P}-E_{P}\right)}{\partial q_{i}}=-\frac{\partial W_{d}}{\partial \dot{q}_{i}}+\frac{\partial W_{e}}{\partial q_{i}}
$$

9 where $q_{i}$ is the generalized displacement and $i$ is the mode number. The form of 10 external load is expressed as follows

$$
F=-F_{0} \cos \left(\Omega t+\theta_{0}\right)
$$

12 where $F_{0}, \Omega$ and $\theta_{0}$ are the excitation amplitude, excitation frequency and

13 phase difference between the axial excitation and the temperature. With the aid of 14 related equations, a system of nonlinear differential equations describing the 15 axisymmetric motion of thin-walled cylindrical shells can be written in the 16 following matrix form

$$
\begin{aligned}
& \mathbf{M} \ddot{\mathbf{q}}+\mathbf{C} \dot{\mathbf{q}}+\left[\mathbf{E}+\boldsymbol{\delta}_{1} \cos \left(\omega_{\mathrm{T}} t\right)\right] \mathbf{K}_{1} \mathbf{q}+\left[\mathbf{E}+\boldsymbol{\delta}_{2} \cos \left(\omega_{\mathrm{T}} t\right)\right] \mathbf{K}_{2}(\mathbf{q}) \mathbf{q} \\
& +\left[\mathbf{E}+\boldsymbol{\delta}_{3} \cos \left(\omega_{\mathrm{T}} t\right)\right] \mathbf{K}_{3}(\mathbf{q}, \mathbf{q}) \mathbf{q}=\mathbf{F} \cos \left(\Omega t+\theta_{0}\right)
\end{aligned}
$$

18 where $\mathbf{E}, \mathbf{M}, \mathbf{C}, \mathbf{K}_{1}, \mathbf{q}$ and $\mathbf{P}$ are the identity matrix, mass matrix,

19 damping matrix, linear stiffness matrix, generalized displacement column vector 20 and load amplitude column vector, respectively. $\mathbf{K}_{2}$ and $\mathbf{K}_{3}$ are quadratic 21 nonlinear stiffness matrix and cubic nonlinear stiffness matrix, which give the 22 quadratic and cubic non-linear stiffness terms associated with displacements. $\boldsymbol{\delta}_{1}$, $23 \boldsymbol{\delta}_{2}$ and $\boldsymbol{\delta}_{3}$ are disturbance parts of stiffness matrix caused by temperature 24 variation. The related matrix dimension is $2 M \times 2 M$, where $M$ is the 25 truncation order.

26 Furthermore, it is convenient to introduce the following dimensionless 27 notations, 


$$
\tau=\omega_{0} t, \eta=\frac{\Omega}{\omega_{0}}, \eta_{\mathrm{T}}=\frac{\omega_{\mathrm{T}}}{\omega_{0}}, \overline{\mathbf{q}}=\frac{1}{h} \mathbf{q}, \overline{\mathbf{F}}=\frac{1}{\omega_{0}^{2} h} \mathbf{M}^{-1} \mathbf{F},
$$

$$
\overline{\mathbf{C}}=\frac{1}{\omega_{0}} \mathbf{M}^{-1} \mathbf{C}, \overline{\mathbf{K}}=\frac{1}{\omega_{0}^{2}} \mathbf{M}^{-1} \mathbf{K}_{1}, \overline{\mathbf{K}}_{2}=\frac{h}{\omega_{0}^{2}} \mathbf{M}^{-1} \mathbf{K}_{2}, \overline{\mathbf{K}}_{3}=\frac{h^{2}}{\omega_{0}^{2}} \mathbf{M}^{-1} \mathbf{K}_{3}
$$

2 where $\omega_{0}$ is the fundamental frequency. Hence, the system (23) is rewritten as

$$
\begin{aligned}
\overline{\mathbf{q}}^{\prime \prime}+ & \overline{\mathbf{C}} \overline{\mathbf{q}}^{\prime}+\overline{\mathbf{K}}_{1} \overline{\mathbf{q}}+\overline{\mathbf{K}}_{2}(\overline{\mathbf{q}}) \overline{\mathbf{q}}+\overline{\mathbf{K}}_{3}(\overline{\mathbf{q}}, \overline{\mathbf{q}}) \overline{\mathbf{q}}=\overline{\mathbf{F}} \cos (\eta \tau) \\
& -\left[\boldsymbol{\delta}_{1} \overline{\mathbf{K}}_{1} \overline{\mathbf{q}}+\boldsymbol{\delta}_{2} \overline{\mathbf{K}}_{2}(\overline{\mathbf{q}}) \overline{\mathbf{q}}+\boldsymbol{\delta}_{3} \overline{\mathbf{K}}_{3}(\overline{\mathbf{q}}, \overline{\mathbf{q}}) \overline{\mathbf{q}}\right] \cos \left(\eta_{\mathrm{T}} \tau-\theta_{0}\right)
\end{aligned}
$$

4 In summary, the axisymmetric nonlinear differential equations describing the 5 motion of the thin-walled cylindrical shell in the time-varying temperature field

6 subjected to the axial excitation have been derived. It is not difficult to find that

7 the system of nonlinear equations present the influences of the parametric

8 excitation and external excitation.

\section{4. Natural frequency analysis}

10 In this paper, the nonlinear vibration of thin-walled shell is considered. The

11 thickness-diameter ratio, the radius and the material density are taken as

$12 h / R=0.02, R=100 \mathrm{~mm}$ and $\rho=1100 \mathrm{~kg} \cdot \mathrm{m}^{-3}$.

\section{$13 \quad 4.1$ Influence of structural parameters on frequency}

14 For thin-walled cylindrical shells, the most important structural parameter is the

15 length-diameter ratio. The influence of this parameter on the natural frequency is 16 analyzed in the following, and the temperature is taken as the room temperature 17 (i.e., 297K).

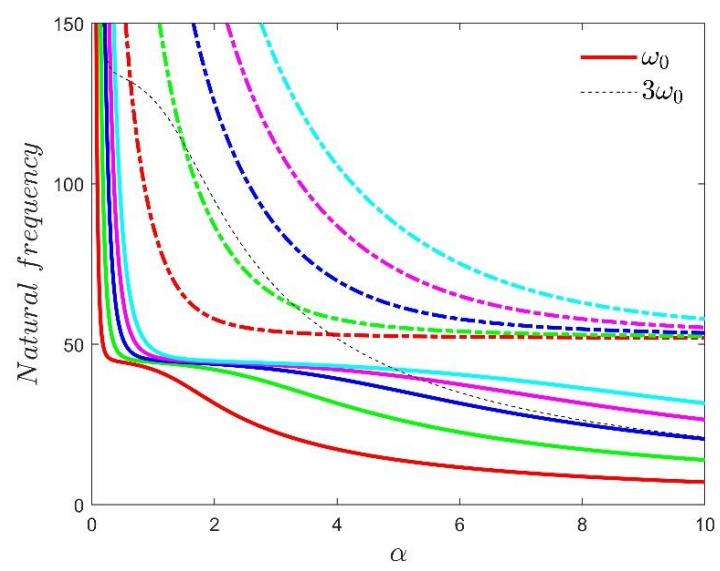


Fig. 2 Relation between the natural frequency and length-diameter ratio (solid line: modes with $m=1 \sim 5$; dash-dotted line: the same order mode with $m=1 \sim 5$ )

Refer to the existing Ref. [33], with the assumption of axisymmetric motion,

4 for the given longitudinal half wave number $m$, there are two modes which can

5 be obtained from the system (23), and denoted the one with lower frequency as

6 the $m$-th order mode, while the other higher as the same order mode of the $m$-th

7 order mode. The solid line in Fig. 2 shows the 1 5th modes from bottom to top. It

8 is not difficult to find that the corresponding low-order mode frequency for $m=1$

9 possesses the fundamental frequency $\omega_{0}$ of the shell (the red solid line). The

10 dash-dotted lines with the same color as the solid lines are the same order modes

11 with the higher natural frequency and the same longitudinal half wave number.

12 For the convenience of later description, the $m$-order mode is denoted as $(m, 1)$,

13 the corresponding same mode is denoted as $(m, 2)$, the corresponding natural

14 frequencies are denoted as $\omega_{m 1}$ and $\omega_{m 2}$. For the fundamental frequency, denote

15 that $\omega_{0}=\omega_{11}$. Moreover, the 3:1 internal resonance is mainly considered in this

16 paper, then the structural parameters satisfying the internal resonance conditions

17 are determined. The intersections of black dashed line and other lines in Fig. 2 are

18 those structural parameters with 3:1 internal resonance, which can be calculated

19 by the dichotomy. As shown in Fig. 2, the natural frequency of the shell is quite

20 sensitive to the variation of structural parameters when the length-diameter ratio is

21 small. That is, in this situation, a small change in the structural parameters will

22 lead to a significant change in the natural frequency, which should result to

23 extremely complex and unexplained internal resonance behavior ${ }^{[33]}$. In order to

24 avoid this problem, this paper will only discuss the larger length-diameter ratio

25 which satisfies the internal resonance condition. There are four structural

26 parameters satisfying the condition and those parameters are greater than 3 , which

27 are listed in Table 2.

28 The internal resonance behavior is very common in various structures, such as 29 typical suspended cables, arches, beams, plates and shells ${ }^{[12,14,34-36]}$. Meanwhile, 30 the internal resonance leads to mode interactions, an energy exchange or a 31 coupling among the modes ${ }^{[37]}$, thus numerous researchers applied it to the energy 32 harvest, and revealed that the working frequency range can be effectively 33 broadened ${ }^{[38-42]}$. 


\begin{tabular}{cc}
\hline Frequency ratios between modes & Valves of $\alpha_{i}$ \\
\hline$\omega_{51} / \omega_{11}=3$ & 4.952848653250840 \\
$\omega_{41} / \omega_{11}=3$ & 5.321955514024012 \\
$\omega_{12} / \omega_{11}=3$ & 3.902195478263820 \\
$\omega_{22} / \omega_{11}=3$ & 3.303421113840159 \\
\hline
\end{tabular}

\section{$3 \quad 4.2$ Effect of temperature on frequency ratio}

4 For the internal resonance behavior of structures, the frequency ratio between the 5 natural frequencies plays a key role. In this paper, the influence of average 6 environmental temperature on the frequency ratio of the shell is examined. The 7 structural parameters are taken as $\alpha=H / D=3.9022, \varepsilon=h / R=0.02$ 。

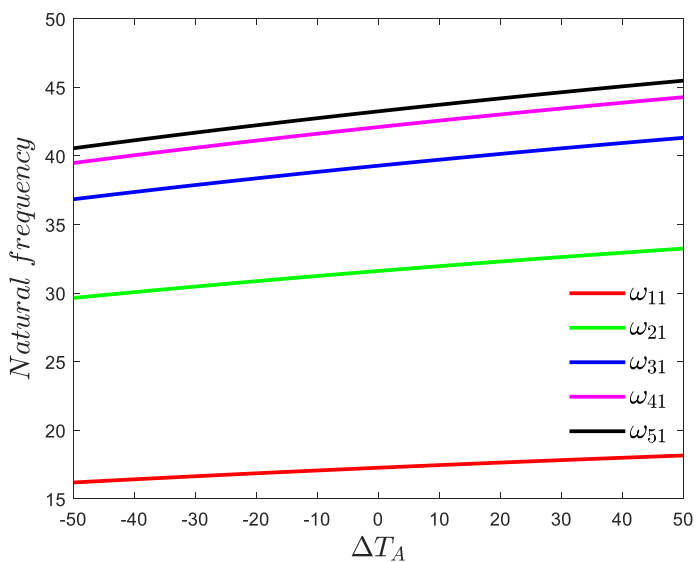

10 Fig 3. Relation between natural frequency and variation of average environmental temperature
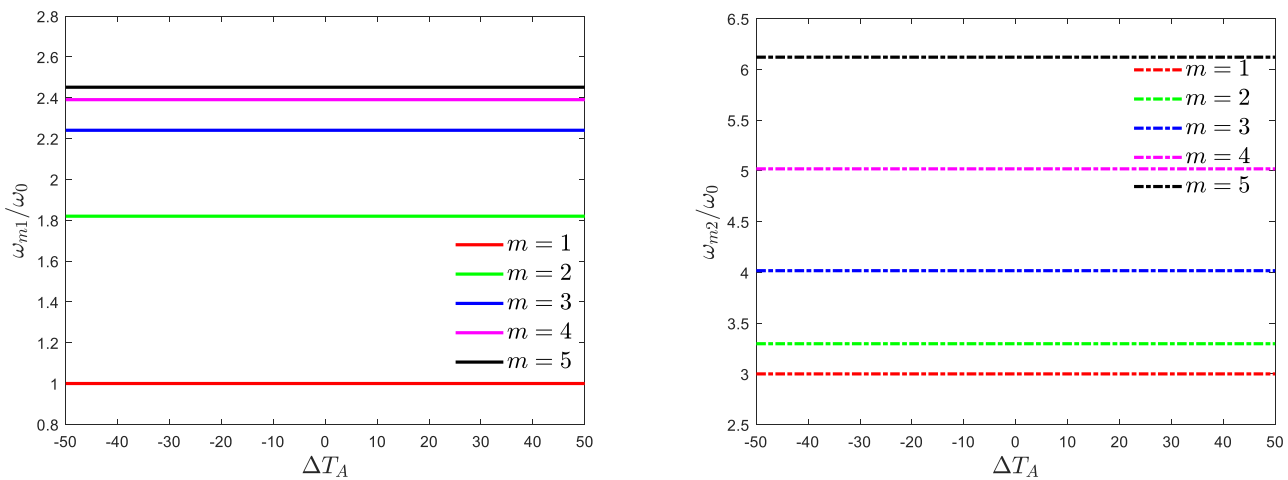

12 Fig. 4 Relation between natural frequency ratio and variation of average environmental 
1 As shown in Fig. 3, the natural frequency increases with the increasing average 2 temperature. The reason is that the constitutive model adopted in this paper 3 gradually hardens as the temperature rises. Moreover, it can be inferred from Eq.

4 (8) that the shear modulus of the material is proportional to the temperature, that 5 is, the higher the environmental temperature, the greater the stiffness, which leads

6 to a greater natural frequency. Meanwhile, it is identified that the variation of 7 average environmental temperature has no effect on the natural frequency ratio, as 8 shown in Fig. 4. Additionally, the internal resonance behavior is very strongly 9 dependent on the natural frequency ratio. Therefore, a preliminarily conclusion 10 can be drawn that the average environmental temperature may not have a 11 significant effect on the axisymmetric nonlinear vibration of the cylindrical shell.

\section{5. Solution}

13 From the foregoing analysis, when the length-diameter ratio equals to the 14 parameters given in Table 2, the mode frequencies of the shell are commensurable 15 or nearly commensurable, which may lead to the internal resonance. Therefore, 16 this article will consider the nonlinear vibration behavior in a periodic uniform 17 temperature field when the parameter is approximately or exactly taken as the 18 values in Table 2.

19 There are three characteristic frequency parameters in Eq. (25), i.e., the 20 fundamental frequency $\omega_{0}=\omega_{11}$, the external excitation frequency $\Omega$, and the 21 periodic uniform temperature frequency $\omega_{\mathrm{T}}$. Equation (24) gives the frequency 22 ratios $\eta=\Omega / \omega_{0}$ and $\eta_{\mathrm{T}}=\omega_{\mathrm{T}} / \omega_{0}$. For the length-diameter ratio parameters 23 listed in Table 2, it is patently obvious that $3 \omega_{0}=\omega_{i}$, where $\omega_{i}$ is the natural 24 frequency of other discrete mode. Since this paper only focuses on the low25 frequency resonance, the frequency should satisfy the requirement that $\Omega \approx \omega_{0}$, 26 i.e., $\eta \approx 1$.

27 It is not difficult to find that Eq. (25) is a system of nonlinear differential 28 equations with quadratic and cubic nonlinear terms. For this kind of equations, the 29 analytical method usually fails, and the common strategy is to adopt the numerical 
1 integration method or the approximate analytical method. Moreover, in order to

2 reveal the influence of structural parameters, the variation should result to some

3 stiff equations which leads to extremely high computational effort or low

4 accuracy. Fortunately, the approximate analytical methods overcome these

5 shortcomings. For the case that only the steady-state periodic solution is required,

6 the harmonic balance method is an extremely excellent choice. In the field of the

7 research on the nonlinear dynamic system, Nayfeh and Mook ${ }^{[43]}$ are the pioneers

8 who adopt the harmonic balance method, and a more systematic introduction to

9 this method can be found in the works of Krack and Gross ${ }^{[44]}$.

10 Employing the harmonic balance method to solve Eq. (25), it is necessary to 11 assume that the solutions of the Eq. (25) can be approximated by the following 12 Fourier series

$$
\left\{\begin{array}{l}
U_{k 0}=A_{01}^{(k)}+\sum_{j=1}^{N}\left[B_{j 1}^{(k)}(t) \cos (j \eta \tau)+C_{j 1}^{(k)}(t) \sin (j \eta \tau)\right], \\
W_{k 0}=A_{02}^{(k)}+\sum_{j=1}^{N}\left[B_{j 2}^{(k)}(t) \cos (j \eta \tau)+C_{j 2}^{(k)}(t) \sin (j \eta \tau)\right]
\end{array}\right.
$$

$$
A_{0 i}^{(k)}=A_{0 i}^{(k)}, A_{j i}^{(k)}=\sqrt{B_{j i}^{(k) 2}+C_{j i}^{(k) 2}}, i=1,2 ; j=1,2, \cdots, N
$$

15 where $k$ is the longitudinal half wave number, $j$ is the order of harmonic.

$16 i=1$ represents the axial direction, while $i=2$ represents the radial direction.

17 For the investigation of the resonance, the frequencies of external excitation

18 and temperature are both close to the fundamental frequency, i.e., $\omega_{0} \approx \Omega=\omega_{\mathrm{T}}$, 19 and $\eta=\eta_{T} \approx 1$. Moreover, it can be inferred from Eqs. (8) (15) that when the 20 perturbed temperature $\Delta T_{\mathrm{P}}$ equals to zero, the parametric excitation part of Eq. 21 (25) will also be zero. and the parametrically excited vibration degenerates into 22 nonlinear forced vibration, namely

$$
\overline{\mathbf{q}}^{\prime \prime}+\overline{\mathbf{C}}^{\prime} \overline{\mathbf{q}}^{\prime}+\overline{\mathbf{K}}_{1} \overline{\mathbf{q}}+\overline{\mathbf{K}}_{2}(\overline{\mathbf{q}}) \overline{\mathbf{q}}+\overline{\mathbf{K}}_{3}(\overline{\mathbf{q}}, \overline{\mathbf{q}}) \overline{\mathbf{q}}=\overline{\mathbf{F}} \cos (\eta \tau)
$$

24 Substituting Eq. (26) into the corresponding nonlinear differential equations, 25 sorting out the coefficient matrix of different harmonics, and then solving it by the 26 arc length method ${ }^{[33]}$, the periodic solution of the equation can be obtained. 


\section{6. Analysis and discussion}

2 The discrete mode method is used to approximate the nonlinear vibration of the

3 shell, in general, it is necessary to discuss the influence of the discrete mode

4 number on the convergence of the solution.

\section{$5 \quad 6.1$ Influence of discrete mode number}

6 Before analyzing the influence of the discrete mode number, this subsection first

7 analyzes the different influence between odd and even order modes.

8

9

10

11

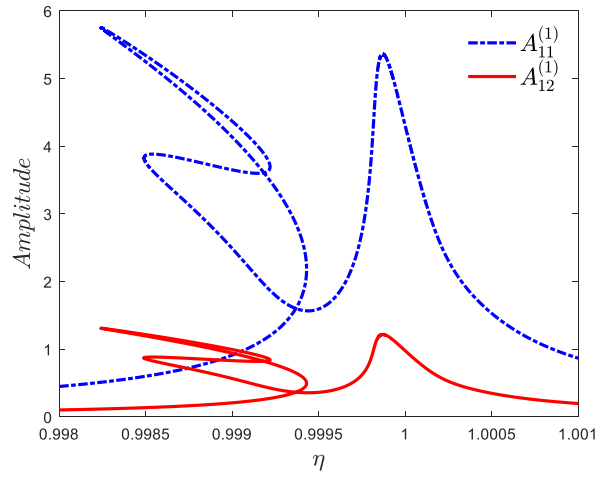

(a)

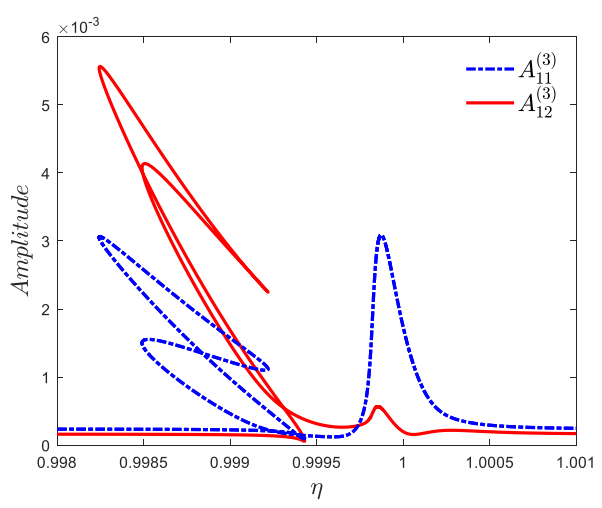

(c)

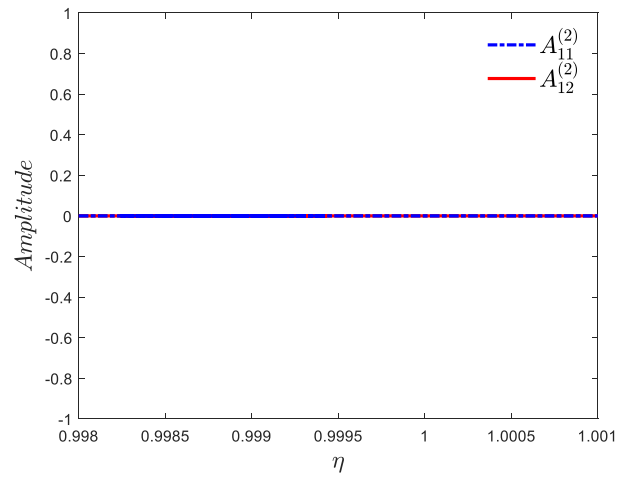

(b)

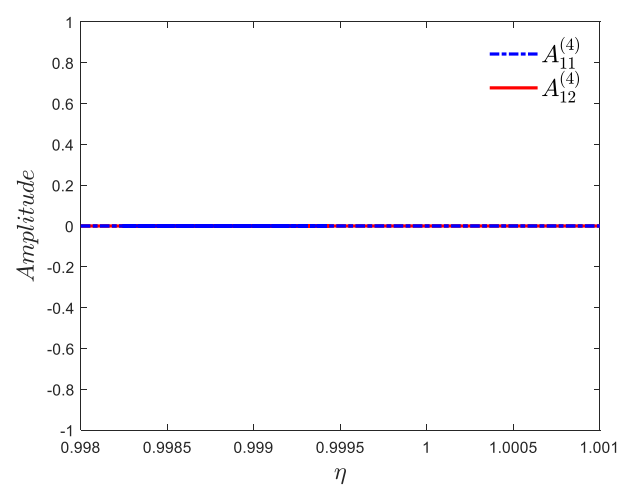

(d) 


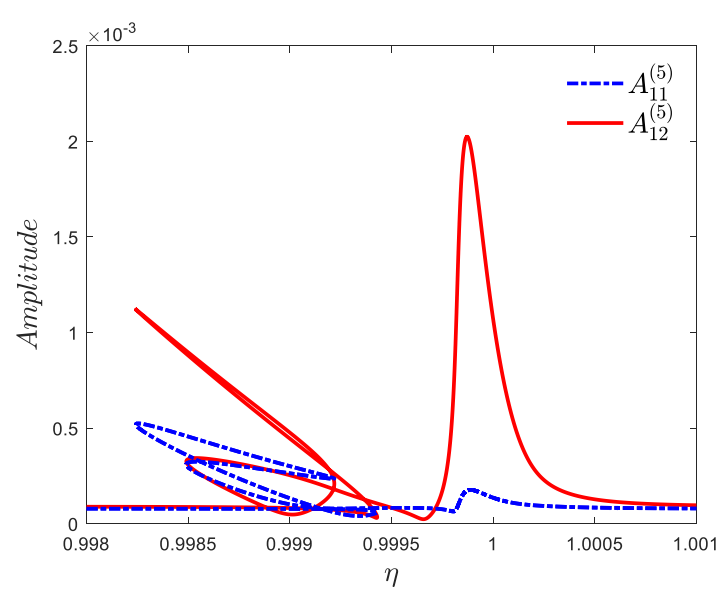

(e)

Fig. 5 Frequency amplitude relationship of 1-order harmonics of different modes for

$$
\alpha=3.9022, \Omega=\omega_{0}=\omega_{12} / 3
$$

5 As shown in Fig. 5, when the axial excitation acting on both ends of the

6 cylindrical shell is symmetrical, the axisymmetric vibration modes with even

7 number would not be excited. It can be clearly seen that when the longitudinal

8 half wave number $m$ is even, the amplitude values given by the frequency

9 amplitude response curve are all zero (as shown in Figs. 5(b) and 5(d)). Thus, the

10 parameters $\alpha_{2}$ and $\alpha_{4}$ given in Table 2 which are the frequency ratio of even

11 order mode to fundamental mode, will not be discussed and analyzed further.

12 Since the internal resonance condition is satisfied or nearly satisfied, it can be

13 observed that there are many nonlinear peaks in the response curve, which reveals

14 the complex energy transfer behavior, and more details in the parameter analysis

15 will be discussed later. In addition, it is not difficult to find that the amplitude

16 magnitude of the high-order mode is far less than that of low-order mode, which

17 indicates that most energy of the shell vibration converges in the lower frequency

18 region. Therefore, for the analysis of shells, it is essential to focus on the low

19 order modes which possess smaller longitudinal half wave number. The case that

20 the parameter equals to $\alpha_{5}$ would not be analyzed further, which means this

21 paper mainly focuses on the case that the structural parameter is somewhere

22 around $\alpha_{3}$. In this situation, the frequency ratio of the fundamental mode to its

23 same order mode satisfies the 3:1 internal resonance condition. 


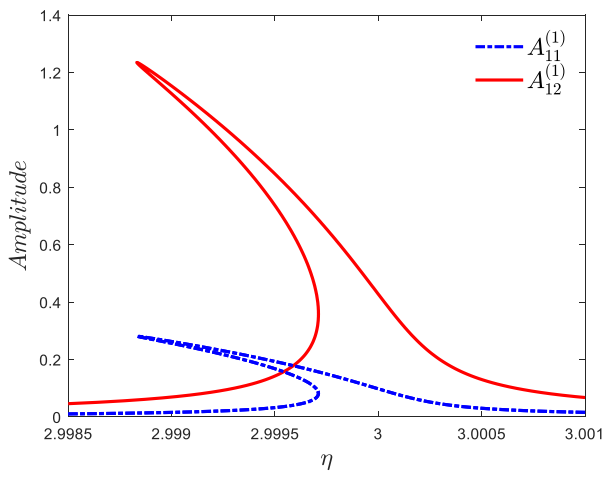

(a)

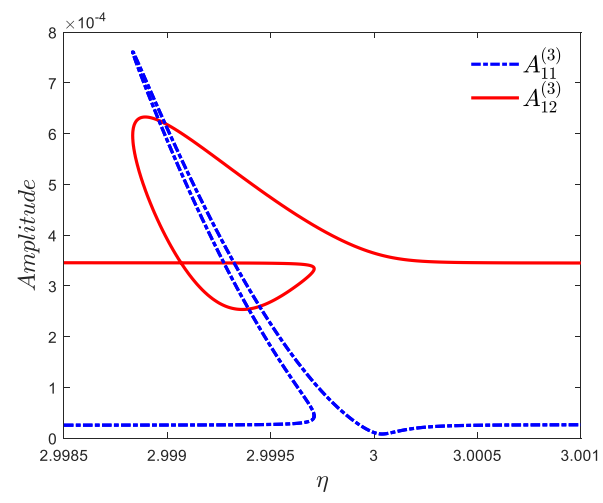

(b)

Fig. 6 Frequency amplitude relationship of 1-order harmonics of different modes with

$$
\alpha=3.9022 \text { and } \Omega=\omega_{12}=3 \omega_{0}
$$

5 As shown in Fig. 5, it can be found that when the axial excitation frequency is 6 close to the fundamental frequency, the low-frequency mode is directly excited, 7 while the high-frequency mode is hardly to be excited. Figure 5(a) clearly shows 8 that the low-order mode which has the largest axial displacement is dominant, 9 while the high-order modes present a very significant difference in the amplitude 10 magnitude. Interestingly, when the external excitation frequency is close to the 11 high-frequency mode, as shown in Fig. 6 (a), it can be found that the response of 12 the low-order mode is no longer dominated by the axial displacement, and the 13 multiple peak phenomenon no longer appears. There is only a left-bent peak 14 which shows the strong softening behavior. The reason is that when the length15 diameter ratio is large, the radial displacement of the high-order mode is dominant $16^{[33]}$, which means the most energy is distributed in the radial direction, and the 17 energy of the high-order mode is easily transferred to the low-order mode. Figure 18 6(a) clearly shows that the radial displacement of low-order mode is largest, 19 which is different from Fig. 5(a).

20 The previous analysis manifests that, for the same computational complexity, to 21 keep only odd order modes in discrete modes is more efficient. Additionally, the 22 influence of the discrete mode number on the structural response is analyzed in 23 the following part. 


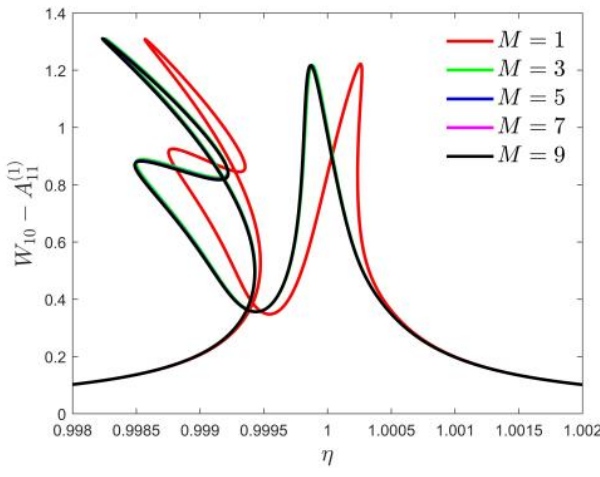

(a)

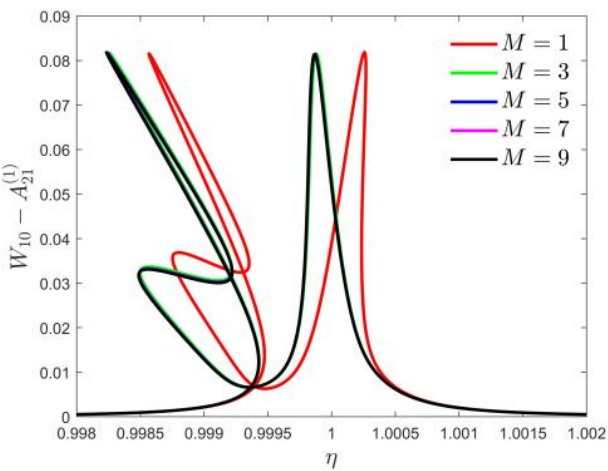

(c)

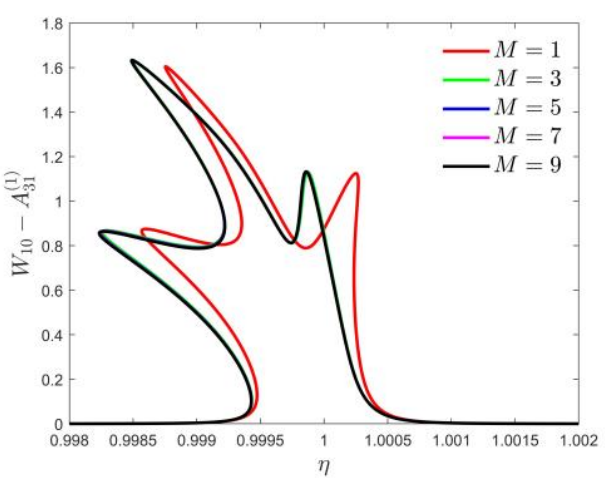

(e)

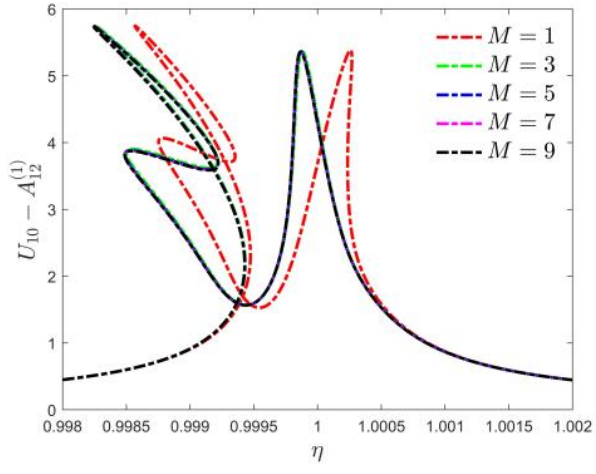

(b)

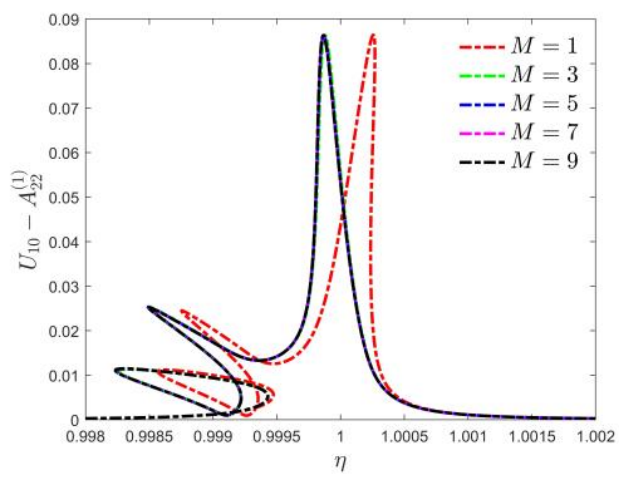

(d)

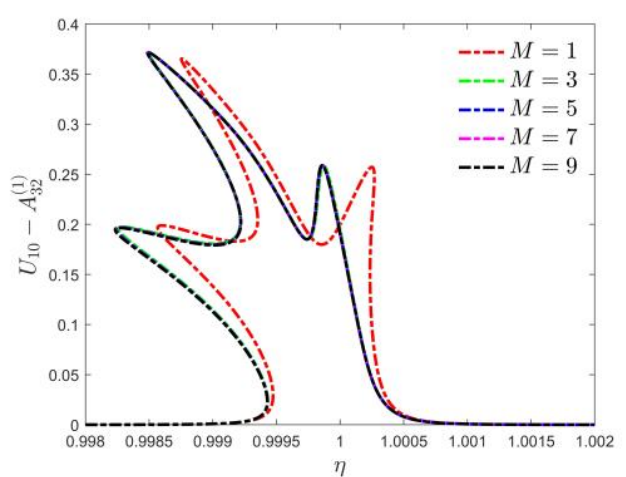

(f)

$7 \quad$ Fig. 7 Influence of discrete mode number on frequency amplitude relationship of different harmonics with $\alpha=3.9022$ and $m=1$

9 It is not difficult to find that the dynamic response of the shell can be accurately

10 described by using two odd order discrete modes, while only one odd order

11 discrete mode should make the response curve tend to be the hardening behavior.

12 Figure 7 also shows that when the condition of 3:1 internal resonance is satisfied

13 approximately, the axial and radial motions of the shell are both dominated by the

14 first and third harmonics which possess similar curve shapes in two directions and

15 the different amplitude magnitude. Additionally, another distinction is that the 
1 second harmonics possess different curve shapes in two directions, and the

2 softening behavior is more obvious for the radial direction. Moreover, the energy

3 distribution between two directions is different, i.e., the most energy of axial

4 direction converges in the first harmonic which is the low frequency region, while

5 the more energy of radial direction converges in the third harmonic which is the

6 high frequency region.

\section{$7 \quad 6.2$ Influence of structural parameters}

8 In this subsection, the value of the length-diameter ratio which satisfy the $3: 1$

9 internal resonance is taken as the critical parameter, i.e., $\alpha=\alpha_{3}$, and the

10 influence of the ratio on the response curve is discussed from the two cases that

11 the ratio gradually decreases or increases. Meanwhile, the stability of the solution

12 is determined, which is based on the eigenvalues of the Jacobian matrix, that is, if

13 the real parts of all eigenvalues are negative, the solution is stable. If there is an

14 eigenvalue with the positive real part, then the solution is unstable ${ }^{[33]}$. As shown

15 in Figs. 8 and 9, the length-diameter ratio is slightly smaller or greater than the

16 critical parameter $\alpha_{3}$, where the dash-dotted lines represent the unstable periodic

17 solution, while the solid line represent the stable periodic solution. Moreover, it is

18 similar to the previous discussion in Fig. 7, i.e., the shell displacement is

19 dominated by the first and third harmonics, while the second harmonics are minor.

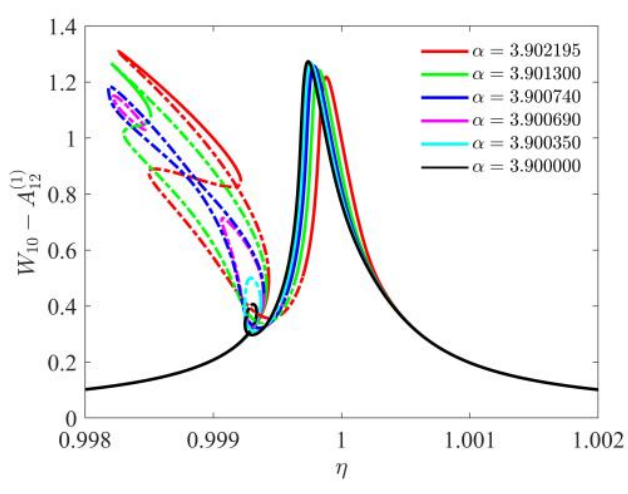

(a)

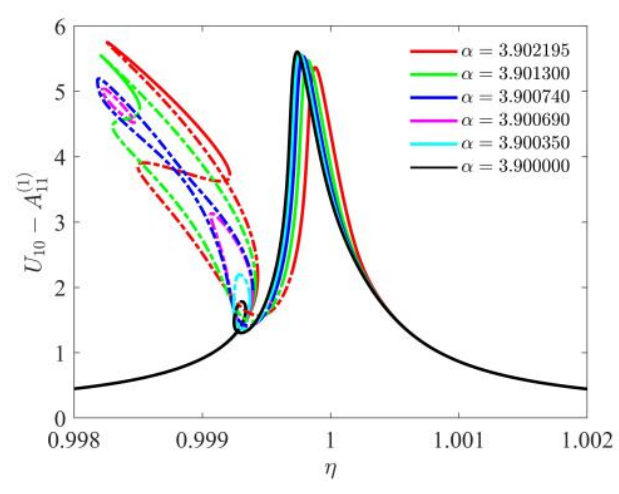

(b) 


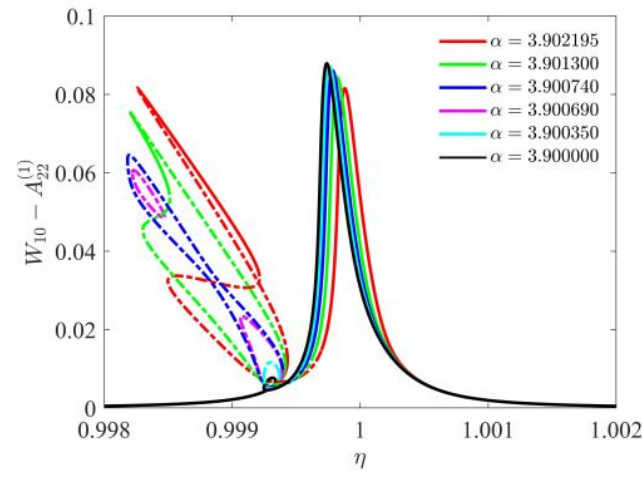

(c)

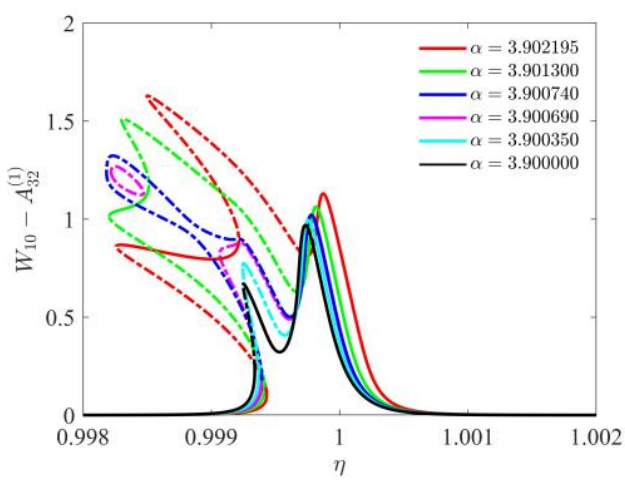

(e)

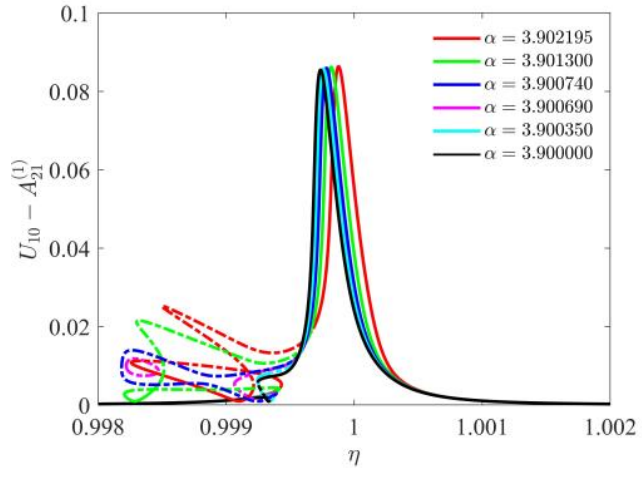

(d)

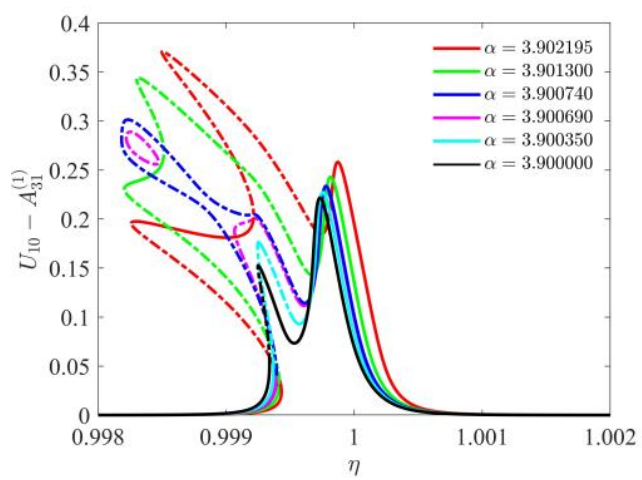

(f)

Fig. 8 Influence of decreasing structural parameters on frequency amplitude relationship

Figure 8 mainly shows the case that the structural parameters are slightly less than the critical parameter $\alpha_{3}$. In this situation, the shell response has three peaks

8 named as the left peak, the middle peak and the right peak respectively, which is 9 more clearly distinguished from Figs. 8(e) and 8(f) (i.e., the third harmonic).

10 Obviously, the left is a typical softening peak with both stable and unstable parts,

11 and there will be a jumping near the frequency where the stability changes. Based

12 on this feature, it is not difficult to infer that for the first and second harmonics,

13 the positions of the left and the middle are exchanged. The middle is a completely 14 unstable peak, which is similar to the case of $2: 1$ internal resonance ${ }^{[45]}$, while the 15 right is a completely stable peak. With the decreasing ratio, the stable region on 16 the right side of the left peak will gradually decrease until it disappears, and the 17 middle peak will gradually shift to the left until merge with the left peak, which 18 will generate a new left peak, however, it is completely unstable. As the parameter 19 decreases further, the new left peak will shrink between the tip and the valley, and 20 generate an isolated bubble response which is completely unstable. As the ratio 21 decreases further, the isolated bubble will gradually shrink until it disappears 
1 completely, and so does the left peak. For this situation, there will be only a

2 completely stable right peak.

3 Additionally, when the length-diameter ratio is in a certain frequency range,

4 there is no stable solution (such as $\alpha=3.900740$, the corresponding frequency

5 range is about [0.9995, 0.9998]), which indicates that the chaotic response may

6 occur in this range. As the ratio decreases, the parameter condition of internal

7 resonance is no longer satisfied gradually, then the frequency domain of response

8 will decrease gradually. It is necessary to state that, during the process of the

9 parameter evolution, the stability of the right peak remains, but the amplitude 10 changes.

11

12

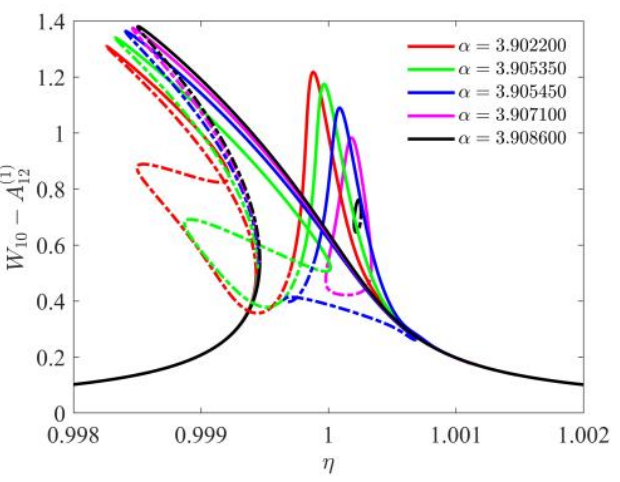

(a)

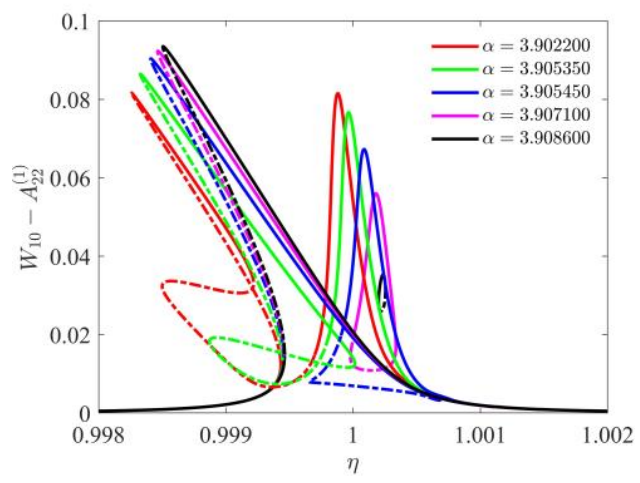

14

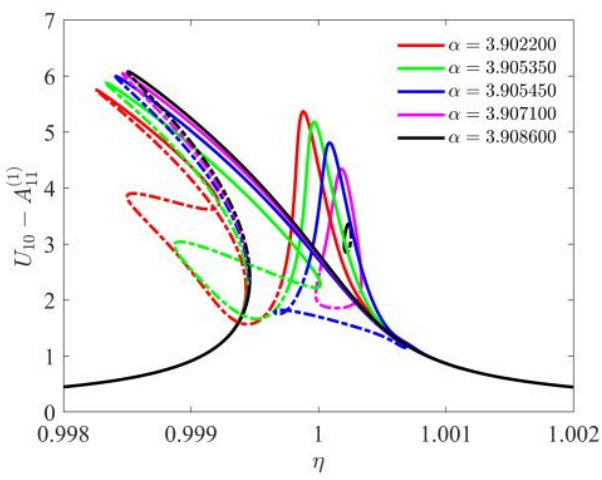

(b)

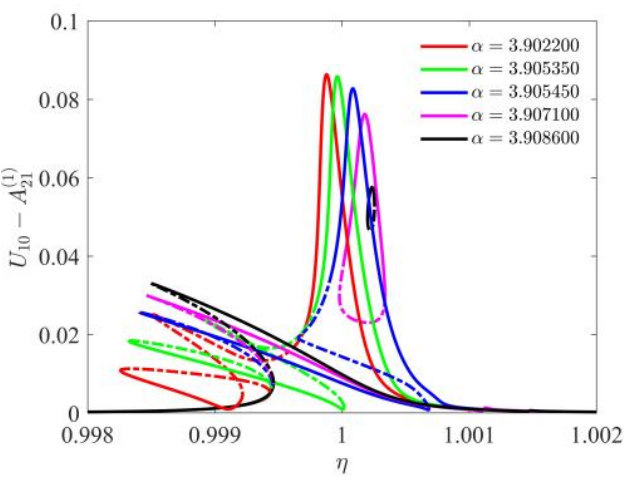

(d)

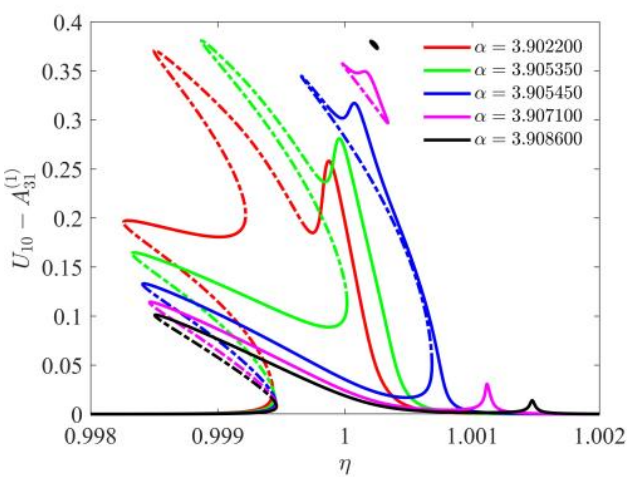


(e)

Fig. 9 Influence of increasing structural parameters on frequency amplitude relationship

3 Figure 9 mainly shows the case that the structural parameters are slightly larger

4 than the critical parameter $\alpha_{3}$. In this situation, the stable region of the left peak

5 will gradually increase with the increasing ratio, and the unstable middle peak will

6 gradually shift to the right and merge with the right peak. As the ratio increases,

7 the middle peak and the right peak will generate an isolated triangular bubble

8 response, where the bottom of the triangle is composed of the unstable middle

9 peak, and most of the others are composed of the stable right peak. Obviously, the

10 evolution process of the 3-order harmonic demonstrates this statement.

11 Meanwhile, since there are stable periodic solutions in the isolated response, there

12 is a phenomenon of coexistence of stable solutions in the corresponding frequency

13 range, that is, one excitation frequency should correspond to two stable periodic

14 solutions, and there are also some jumping phenomena. It is also worth pointed

15 out that for the 1-order and 2-order harmonics, the isolated triangular bubble

16 response may not be obvious due to the existence of the intersection points,

17 however, those triangle bubbles cannot be traced by the arc length method

18 directly, which indicates those bubbles are isolated with the main curves. In order

19 to obtain the isolated bubbles, it is necessary to adopt the strategy mixing the

20 perturbation method and arc length method. As the ratio increases further, the

21 isolated triangular bubble will shrink until it disappears completely. Finally, only

22 the left peak which characterizes the softening remains.

23 In summary, with the increasing ratio, the parameter condition of internal 24 resonance is no longer satisfied gradually, however, it is different from the case of

25 Fig. 8, due to the dominant left peak remains, the frequency domain of response 26 would not decrease significantly.

\section{$27 \quad 6.3$ Influence of temperature parameters}

28 According to Eq. (3), there are three parameters related to temperature, namely 29 the average temperature $T_{\mathrm{A}}$, the perturbed temperature $\Delta T_{\mathrm{P}}$ and the phase 30 difference $\theta_{0}$ between temperature and excitation. The influence of these 31 parameters will be discussed in this subsection. The discussion above mentioned 
1 manifests that the responses are dominated by the first and third order harmonics

2 for the 3:1 internal resonance, and the shapes of the axial and radial response

3 curves are similar, while the amplitude magnitudes are different. Therefore, this

4 subsection only presents the first and third order harmonics of the radial

5 displacement, and the length-diameter ratio is taken as $\alpha=3.902195$.

6

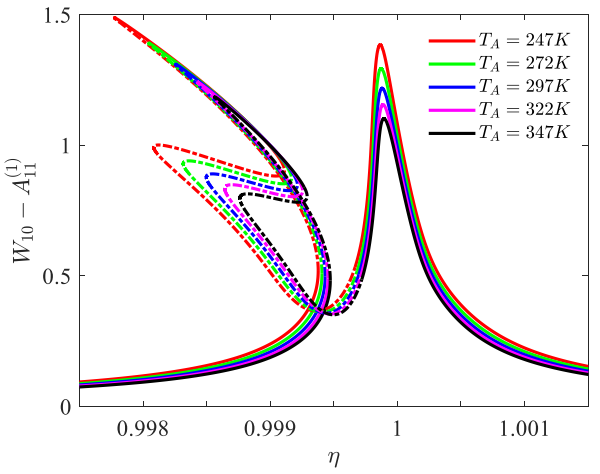

(a)

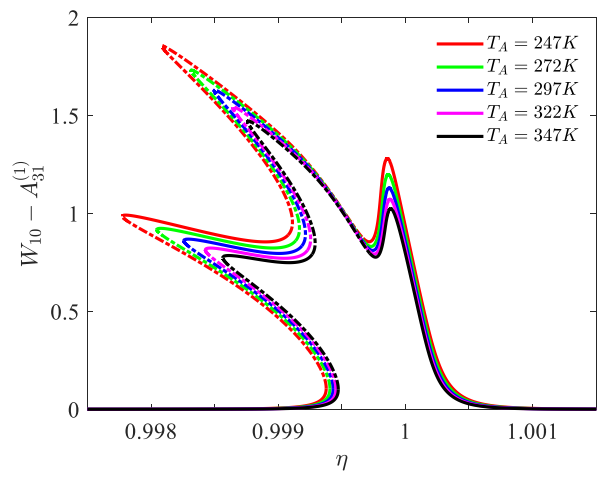

(b)

Fig. 10 Influence of average temperature on frequency amplitude relationship for $\Delta T_{P}=0 \mathrm{~K}$

$$
\text { and } \theta_{0}=0
$$

As shown in Fig. 10, the influence of average temperature on frequency amplitude relationship is presented. Obviously, the higher the average temperature, the smaller the response amplitude. The reason is that the shear modulus is directly proportional to temperature which is shown in Eq. (8). Thus, the higher the temperature, the greater the shear modulus, which means the greater the stiffness of the shell, and the stronger the resistible capacity to the deformation, i.e., the smaller the response amplitude.

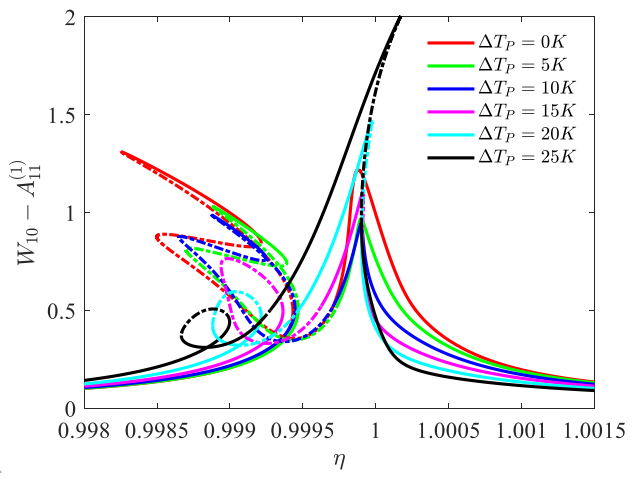

(a)

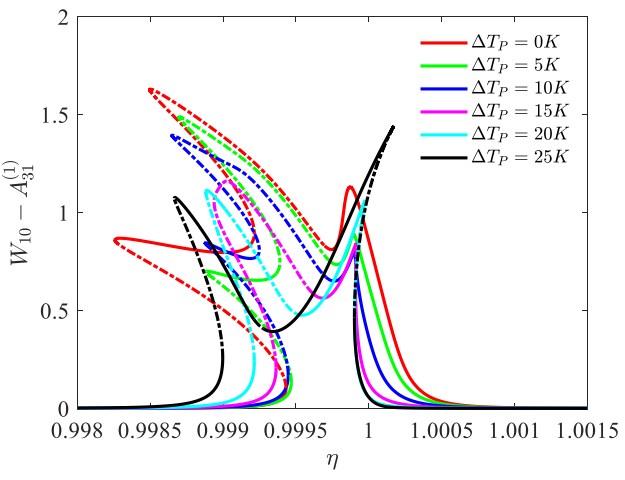

(b)

Fig. 11 Influence of perturbed temperature on frequency amplitude relationship for $T_{A}=297 \mathrm{~K}$, 
1 Figure 11 shows the influence of the perturbed temperature on the vibration

2 response. With the increasing perturbed temperature, the middle peak will 3 gradually distorted and merges with the left peak, which is kind of similar to the 4 situation with gradually decreasing length-diameter ratio (as shown in Fig. 8).

5 However, there is no bubble response during the evolution process, and the right

6 peak will change more significantly. Meanwhile, the softening characteristics of

7 the cylindrical shell will be suppressed gradually with the increasing perturbed

8 temperature, while the hardening characteristics will be enhanced significantly,

9 which is more obvious for the first harmonic. When the perturbed temperature is 10 large enough (for example, $\Delta T_{P}=25 K$, the black line in Fig. 11), the

11 hyperelastic thin-walled cylindrical shell will present a typical hardening peak.

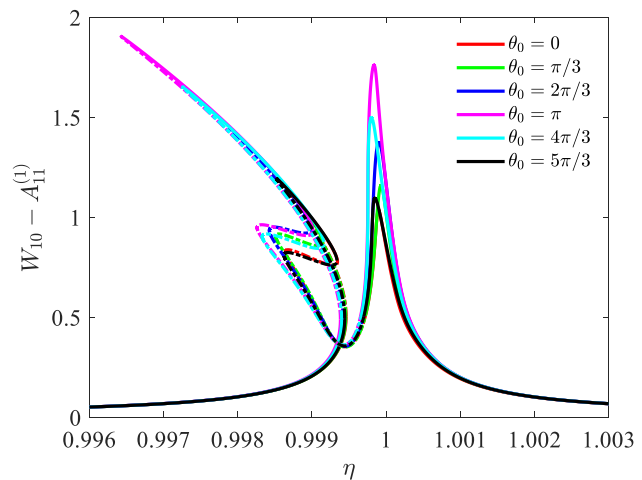

(a)

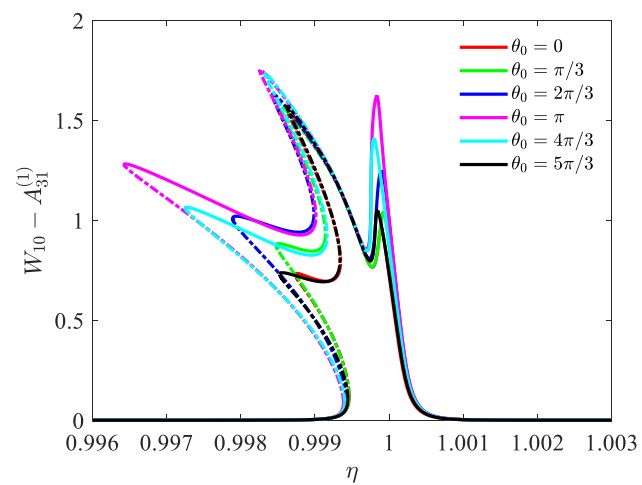

(b)

14 Fig. 12 Influence of phase difference on frequency amplitude relationship for $\Delta T_{P}=0 \mathrm{~K}$,

$$
T_{A}=297 \mathrm{~K}
$$

16 Figure 12 mainly shows the influence of the phase difference between the 17 perturbed temperature and the external excitation on the shell response. 18 Obviously, when the phase difference is $\pi$, the response presents the largest 19 amplitude (the magenta lines), and the amplitude of the three peaks are enhanced 20 significantly. That is, the shell response can be adjusted by controlling the phase 21 difference between the excitation and the temperature.

22 In this subsection, it can be found that the influence of temperature parameters 23 on the response is similar to that of the length-diameter ratio. However, the 24 hardening characteristic caused by the increasing temperature makes some 25 difference. The frequency amplitude response curve evolutes more smoothly with 26 the structural parameter compared with the temperature parameter. Thus, the 
1 isolated bubbles only occur during the evolution process of the structural 2 parameter. Comparing Fig. 8(a) and Fig. 11(a), the evolution behavior of

3 frequency amplitude curve manifests that the temperature (especially the

4 perturbed temperature) will distort the response curve and lead to a more irregular

5 result. Meanwhile, the distortion will enhance the hardening significantly.

6 Briefly, the variation of the structural parameter and the temperature both play

7 an important role in the shell response, they bear relations as well as distinctions.

8 Therefore, it is necessary and essential to conduct the comprehensive analysis,

9 which leads to a better understanding and prediction for the nonlinear dynamic

10 behavior of the hyperelastic thin-walled cylindrical shell.

\section{$11 \quad$ 7. Conclusions}

12 In this paper, the axisymmetric nonlinear vibration of the hyperelastic thin-walled

13 shell is investigated by the harmonic balance method and the arc length method,

14 and the 3:1 internal resonance of the shell is analyzed. Firstly, the system of

15 nonlinear governing differential equations with time-varying parameters are

16 derived based on the variational method. Then the periodic solution is calculated

17 via using the harmonic balance method and the arc length method, and the

18 stability is determined. Finally, the influences of structure and temperature

19 parameters on the evolution of nonlinear vibration are discussed. The mainly

20 conclusions are drawn as follows:

21 (1) For axisymmetric nonlinear vibrations of hyperelastic thin-walled cylindrical

22 shells, it is inaccurate to carry out the analysis with a single mode. Meanwhile,

23 since the even order modes will not be excited, it is more effective to discretize

24 the model with only the odd order mode.

25 (2) For hyperelastic thin-walled cylindrical shells with 3:1 internal resonance, the

26 length-diameter ratio has an extremely important impact on the shell resonance.

27 The reason is that this parameter plays a critical role in the frequency ratio which

28 is the necessary condition for internal resonance. Near the internal resonance

29 parameters, there are abundant nonlinear dynamic behaviors, including the 
1 jumping, softening and hardening, and isolated bubble response. Specially, the left

2 peak will gradually shrink, and the isolated bubble occurs when the decreasing

3 length-diameter ratio is smaller than the critical parameter; while the right peak

4 will gradually shrink, and the isolated bubble occurs when the increasing length-

5 diameter ratio is larger than the critical parameter.

6 (3) The influence of temperature parameters on the nonlinear dynamic behavior of

7 the shell is similar to that of the length-diameter ratio. However, the bubble will

8 not occurs during the temperature parameter varies. In addition, the perturbed

9 temperature has a more obvious effect on the stability of the solution.

10 Acknowledgements

11 This work was supported by the National Natural Science Foundation of China (Nos. 11672069,

$1211672062,11872145,11902068$ ), the Natural Science Foundation of Liaoning Province (grant

13 numbers 2020-BS-077), and the Programme of Introducing Talents of Discipline to Universities

14 Project (No. B08014).

\section{Compliance with ethical standards}

\section{Conflict of interest}

17 The authors declare that they have no conflict of interests.

\section{Data availability statement}

19 All data included in this study are available upon request by contact with the corresponding author.

\section{References}

21 [1] Zhang, W., Zheng, Y., Liu, T., Guo, X.Y.: Multi-pulse jumping double-parameter chaotic 22 dynamics of eccentric rotating ring truss antenna under combined parametric and external excitations. Nonlinear Dynamics 98, 761-800 (2019). 10.1007/s11071-019-05227-8

24 [2] Liu, T., Zhang, W., Mao, J.J., Zheng, Y.: Nonlinear breathing vibrations of eccentric 25 rotating composite laminated circular cylindrical shell subjected to temperature, rotating speed and 26 external excitations. Mechanical Systems and Signal Processing 127, 463-498 (2019). 27 10.1016/j.ymssp.2019.02.061

28 [3] Shariyat, M., Asgari, D.: Nonlinear thermal buckling and postbuckling analyses of 29 imperfect variable thickness temperature-dependent bidirectional functionally graded cylindrical 30 shells. International Journal of Pressure Vessels and Piping 111-112, 310-320 (2013). 31 10.1016/j.ijpvp.2013.09.005 
[4] Jakus, A.E., Rutz, A.L., Jordan, S.W., Kannan, A., Mitchell, S.M., Yun, C., Koube, K.D., Yoo, S.C., Whiteley, H.E., Richter, C.-P., Galiano, R.D., Hsu, W.K., Stock, S.R., Hsu, E.L., Shah, R.N.: Hyperelastic "bone": A highly versatile, growth factor-free, osteoregenerative, scalable, and surgically friendly biomaterial. Science Translational Medicine 8, 358ra127 (2016).

[5] Amabili, M., Balasubramanian, P., Bozzo, I., Breslavsky, I.D., Ferrari, G.: Layer-specific hyperelastic and viscoelastic characterization of human descending thoracic aortas. Journal of the Mechanical Behavior of Biomedical Materials 99, 27-46 (2019). 10.1016/j.jmbbm.2019.07.008

[6] Soedel, W.: Vibrations of Shells and Plates. CRC Press (2004)

[7] Radwanska, M., Stankiewicz, A., Wosatko, A., Pamin, J.: Plate and Shell Structures : Selected Analytical and Finite Element Solutions. John Wiley and Sons (2017)

[8] Reddy, J.N.: Mechanics of Laminated Composite Plates and Shells: Theory and Analysis. CRC Press (2003)

[9] Shen, H.S.: Functionally graded materials - nonlinear analysis of plates and shells. CRC Press (2009)

[10] Shen, H.S.: A two-step perturbation method in nonlinear analysis of beams, plates and shells. John Wiley and Sons (2013)

[11] Amabili, M.: Nonlinear Mechanics of Shells and Plates in Composite, Soft and Biological Materials. Cambridge University Press (2018)

[12] Ye, C., Wang, Y.Q.: Nonlinear forced vibration of functionally graded graphene plateletreinforced metal foam cylindrical shells: internal resonances. Nonlinear Dynamics 1-19 (2021). 10.1007/s11071-021-06401-7

[13] Zhang, W., Yang, S.W., Mao, J.J.: Nonlinear radial breathing vibrations of CFRP laminated cylindrical shell with non-normal boundary conditions subjected to axial pressure and radial line load at two ends. Composite Structures 190, 52-78 (2018). 10.1016/j.compstruct.2018.01.091

[14] Yang, S.W., Zhang, W., Mao, J.J.: Nonlinear vibrations of carbon fiber reinforced polymer laminated cylindrical shell under non-normal boundary conditions with 1:2 internal resonance. European Journal of Mechanics - A/Solids 74, 317-336 (2019). 10.1016/j.euromechsol.2018.11.014

[15] Vuong, P.M., Duc, N.D.: Nonlinear vibration of FGM moderately thick toroidal shell segment within the framework of Reddy's third order-shear deformation shell theory. International Journal of Mechanics and Materials in Design 16, 245-264 (2019). 10.1007/s10999-019-09473-X

[16] Parvez, M.T., Khan, A.H., Yasin, M.Y.: On the softening and hardening nonlinear behavior of laminated cylindrical shells. Engineering Structures 226, 111339 (2021). 10.1016/j.engstruct.2020.111339

[17] Shen, H.S., Li, C., Reddy, J.N.: Large amplitude vibration of FG-CNTRC laminated cylindrical shells with negative Poisson's ratio. Computer Methods in Applied Mechanics and Engineering 360, 112727 (2020). 10.1016/j.cma.2019.112727

39 [18] Wang, R., Ding, H., Yuan, X., Lv, N., Chen, L.: Nonlinear singular traveling waves in a 40 slightly compressible thermo-hyperelastic cylindrical shell. Nonlinear Dynamics (2021). 41 10.1007/s11071-021-06394-3

42 [19] Soares, R.M., Gonçalves, P.B.: Nonlinear vibrations and instabilities of a stretched 43 hyperelastic annular membrane. International Journal of Solids and Structures 49, 514-526 (2012). 44 10.1016/j.ijsolstr.2011.10.019

45 [20] Soares, R.M., Gonçalves, P.B.: Nonlinear vibrations of a rectangular hyperelastic 46 membrane resting on a nonlinear elastic foundation. Meccanica 53, 937-955 (2017). 47 10.1007/s11012-017-0755-5

48 [21] Zhao, Z., Zhang, W., Zhang, H., Yuan, X.: Some interesting nonlinear dynamic behaviors 49 of hyperelastic spherical membranes subjected to dynamic loads. Acta Mechanica 230, 3003-3018 50 (2019). 10.1007/s00707-019-02467-y

51 [22] Chen, W., Wang, L., Dai, H.L.: Nonlinear Free Vibration of Hyperelastic Beams Based 52 on Neo-Hookean Model. International Journal of Structural Stability and Dynamics 20, 2050015 53 (2019). 10.1142/s0219455420500157

54 [23] Wang, Y.B., Ding, H., Chen, L.Q.: Nonlinear vibration of axially accelerating 55 hyperelastic beams. International Journal of Non-Linear Mechanics 99, 302-310 (2018). 56 10.1016/j.ijnonlinmec.2017.12.013

57 [24] Wang, Y.B., Ding, H., Chen, L.Q.: Vibration of axially moving hyperelastic beam with 58 finite deformation. Applied Mathematical Modelling 71, 269-285 (2019). 59 10.1016/j.apm.2019.02.011 
[25] Dong, L., Grissom, M.D., Prasad, M.G., Fisher, F.T.: Application of mechanical stretch to tune the resonance frequency of hyperelastic membrane-based energy harvesters. Sensors and Actuators A - Physical 252, 165-173 (2016). 10.1016/j.sna.2016.10.034

[26] Wang, Y.B., Zhu, W.D.: Nonlinear transverse vibration of a hyperelastic beam under harmonic axial loading in the subcritical buckling regime. Applied Mathematical Modelling 94, 597-618 (2021). 10.1016/j.apm.2021.01.030

[27] Aranda-Iglesias, D., Martínez, J.A.R., Rubin, M.B.: Nonlinear axisymmetric vibrations of a hyperelastic orthotropic cylinder. International Journal of Non-Linear Mechanics 99, 131-143 (2018). 10.1016/j.ijnonlinmec.2017.11.007

[28] Alibakhshi, A., Heidari, H.: Nonlinear dynamics of dielectric elastomer balloons based on the Gent-Gent hyperelastic model. European Journal of Mechanics - A/Solids 82, 103986 (2020). 10.1016/j.euromechsol.2020.103986

[29] Breslavsky, I.D., Amabili, M., Legrand, M.: Static and Dynamic Behavior of Circular Cylindrical Shell Made of Hyperelastic Arterial Material. Journal of Applied Mechanics Transactions of the ASME 83, 051002 (2016). 10.1115/1.4032549

[30] Amabili, M., Balasubramanian, P., Breslavsky, I.D., Ferrari, G., Garziera, R., Riabova, K.: Experimental and numerical study on vibrations and static deflection of a thin hyperelastic plate. Journal of Sound and Vibration 385, 81-92 (2016). 10.1016/j.jsv.2016.09.015

[31] Arruda, E.M., Boyce, M.C.: A three-dimensional constitutive model for the large stretch behavior of rubber elastic materials. Journal of the Mechanics and Physics of Solids 41, 389-412 (1993). https://doi.org/10.1016/0022-5096(93)90013-6.

[32] Xia, Y.M., Li, Z.R., Dong, Y., Li, X.: Experimental Study on the Temperature Dependence of Hyperelastic Behavior of Tire Rubbers under Moderate Finite Deformation. Rubber Chemistry and Technology 84, 215-228 (2011). 10.5254/1.3577534

[33] Xu, J., Yuan, X.G., Jiao, J., Zhang, H.W.: Computation of axisymmetric nonlinear lowfrequency resonances of hyperelastic thin-walled cylindrical shells. Applied Mathematical Modelling 94, 332-349 (2021). 10.1016/j.apm.2021.01.027

[34] Christopher L. Lee , N.C.P.: Nonlinear oscillations of suspended cables containing a twoto-one internal resonance. Nonlinear Dynamics 3, 465-490 (1992).

[35] Hajjaj, A.Z., Alfosail, F.K., Younis, M.I.: Two-to-one internal resonance of MEMS arch resonators. International Journal of Non-Linear Mechanics 107, 64-72 (2018). 10.1016/j.ijnonlinmec.2018.09.014

[36] Jiang, W.A., Chen, L.Q., Ding, H.: Internal resonance in axially loaded beam energy harvesters with an oscillator to enhance the bandwidth. Nonlinear Dynamics 85, 2507-2520 (2016). 10.1007/s11071-016-2841-y

[37] Manevich, A.I., Manevitch, L.I.: The mechanics of nonlinear systems with internal resonances. Imperial College Press (2005)

[38] Chen, L.Q., Jiang, W.A.: Internal Resonance Energy Harvesting. Journal of Applied Mechanics - Transactions of the ASME 82, 031004 (2015). 10.1115/1.4029606

[39] Yang, W., Towfighian, S.: Internal resonance and low frequency vibration energy harvesting. Smart Materials and Structures 26, 095008 (2017). 10.1088/1361-665X/aa791d

[40] Xiong, L.Y., Tang, L.H., Mace, B.R.: A comprehensive study of 2:1 internal-resonancebased piezoelectric vibration energy harvesting. Nonlinear Dynamics 91, 1817-1834 (2018). 10.1007/s11071-017-3982-3

[41] Jiang, W.N., Han, X.J., Chen, L.Q., Bi, Q.S.: Improving energy harvesting by internal resonance in a spring-pendulum system. Acta Mechanica Sinica 36, 618-623 (2020). 10.1007/s10409-020-00945-4

[42] Jiang, W.A., Ma, X.D., Han, X.J., Chen, L.Q., Bi, Q.S.: Broadband energy harvesting based on one-to-one internal resonance. Chinese Physics B 29, (2020). 10.1088/1674-1056/aba5fd [43] Nayfeh, A.H., Mook, D.T.: Nonlinear oscillations. Wiley (1995)

[44] Krack, M., Gross, J.: Harmonic Balance for Nonlinear Vibration Problems. Springer International Publishing (2019)

[45] Chen, L.Q., Zhang, G.C., Ding, H.: Internal resonance in forced vibration of coupled cantilevers subjected to magnetic interaction. Journal of Sound and Vibration 354, 196-218 (2015). 10.1016/j.jsv.2015.06.010 